\title{
INTRODUCTION AND INVESTIGATION OF A METHOD FOR THE SYNTHESIS AND SELECTION OF HYBRID ELECTRIC VEHICLE DRIVETRAIN DESIGN IN THE EARLY STAGE OF PRODUCT GENERATION ENGINEERING (PGE)
}

\author{
A. Albers ${ }^{\otimes}$, S. Ruoff and K. Bause \\ Karlsruhe Institute of Technology, Germany \\ $\bowtie$ sekretariat@ipek.kit.edu
}

\begin{abstract}
In this paper, a method is presented, that supports the developer in the development of hybrid electrical vehicle drivetrains. The developer gets support in the early stage of product generation engineering, beginning with the definition of the system of objectives, via the automated synthesis and investigation of the drivetrain topologies, ending with a topology ranking, which is related to the system of objectives, and a similarity analysis. As the method is implemented in a tool, the results of the tool and its usability are investigated within two comprehensive descriptive studies.
\end{abstract}

Keywords: design tools, modelling, complex systems, automated rating

\section{Introduction}

Higher restrictions on vehicle emissions force the vehicle manufacturers to make their drivetrains more efficient. Besides downsizing measures of the internal combustion engine (ICE) and exhaust cleaning systems, the electrification of the drivetrain is in focus since years to fulfil the fleet emission limits. As customers struggle with the current disadvantages, as low electric range, not enough charger stations, long charging times and high costs, of pure electric vehicles (EV), hybrid electric vehicles (HEV) are in the focus of the drivetrain development. HEVs combine the advantages of conventional and electric vehicles, as they provide a long driving range, fast fuelling and local emission free driving.

As the complexity of the drivetrain is rising with the number of subcomponents within it, the developer has to handle more ambitious development tasks and needs therefore methodical support. There are various problems within the development of the hybrid drivetrain. The developer has to define the drivetrain topology, which in this context describes the mechanical architecture and interconnections of the drivetrain components. In doing so, the developer must ensure that the specified objectives can be achieved with the defined topology and the given drive units and energy storage systems. Beside the topology of the drivetrain, the applied control strategy plays a key role, as it decides at each driving manoeuver, which driving mode is chosen and which drive units provide the power to accelerate the vehicle. According to Albers et al. (2014), it is not possible to define a control strategy without knowledge about the drivetrain architecture, but without control strategy, the investigation of the efficiency cannot be performed properly. Therefore, a generalized control strategy, which is described in section 4.3, is introduced in order to investigate various topologies in the simulation. Reuschlé et al. 
(2016) presented a method for the development of Plug-In HEV powertrains for the optimization of given topologies. As the amount of mechanical realizable topologies is rising with the amount of subsystems in the drivetrain, the developer has furthermore to investigate a huge number of drivetrain topologies that have to be investigated and rated, in order to identify the most suitable drivetrain for the defined system of objectives. The high amount of drivetrains, which can be more than 800 with one ICE, three electric machines (EMs) and two transmissions, and the challenge with the control strategy cannot be handled manually anymore. Therefore, a method will be presented, that supports the developer within the activities of "detecting ideas", "modelling principle solution and embodiment" and "validate and verify" according to the integrated product engineering model (iPeM) (Albers et al., 2016a).

\section{State of research}

New drivetrain topologies are investigated in the early phase of the product generation development. The early phase begins with the initiation of a project and ends with a rated technical solution, according to Albers et al. (2017). In the context of product generation engineering (PGE), new product generations are developed based on reference products. The development of a new product generation is defined as the combination of subsystems that are developed with carryover, embodiment and principle variation (Albers et al., 2016b).

As carryover and embodiment variation are not as complex as principle variation, there are many approaches that focus on the improvement of single drivetrain components, using carryover variation of the drivetrain topology (Teuschl, 2009, Voss, 2005). Others optimize known drivetrain topologies as a whole system, regarding the subsystems interaction (Eghtessad et al., 2015; Müller et al., 2016; Silvas et al., 2016).

There are only few approaches published for modelling hybrid electric vehicle drivetrains mathematically in order to identify drivetrain structures and to investigate them regarding their efficiency and performance (Sturm et al., 2016; Silvas et al., 2015; Haag, 2017). All of these approaches have in common, that they use the graph theory for modelling the hybrid drivetrains, whereby Haag (2017) combines the description of couplings with the investigation of the driving modes to be realized. Sturm et al. (2016) describes an approach that allows the user to define vehicle parameters and requirements. Based on this input, he defines functions, that have to be fulfilled and searches for topologies that are able to fulfil these functions. His search field is limited, as he only applies one electric motor and one electric generator in his approach. In the presented model of Silvas et al. (2015), which is applicable to all hybrid electric topologies and allows to describe them mathematically, the system of objectives is not clearly described and it is only a model for mathematic modelling of drivetrains and no development approach presented. The developer gets support in the activities of modelling principle solutions and embodiment but he cannot influence the system of objectives, which means that the identified topologies might not be suitable for the use case, except the approach of Sturm et al. (2016). As mentioned in the introduction, the control strategy plays also an important role and is not considered in these approaches, as they mainly focus on the topology description. The methodical support of the developer is not in the focus of the presented approaches, although he has to apply them to a development process. It is also not described in detail how the investigation of the topologies regarding their mechanical and functional suitability is performed.

\section{Research gap and research design}

In section 2, three approaches have been introduced, that allow the mathematical description of HEV drivetrains and their investigation. None of them is focused on the developer and its support needs in the early stage of PGE. There are also methods published that assist the developer in identifying and defining new systems of objectives. The research gap was found in the combination of these methods and the applicability for the developer. There is no single whole method framework to assist the designer in the whole process from identifying and defining the system of objectives for a new generation of HEV drivetrains, via synthesizing new HEV drivetrains, to finally examining them to select the most appropriate drivetrain for the defined system.

The research design is based on the design research methodology approach of Blessing and Chakrabarti (2009). 
Based on the research clarification, which was shortly introduced in section 3, and the presented research gap, a review based descriptive study was performed that figured out the need for a method for the support of the developer in the early stage of PGE of HEVs.

The aim of the research is therefore, to provide a method, which supports the developer within the product engineering process within the activities of "detecting ideas", "modelling principal solutions and embodiment" and "validation and verification".

The new method shall support the developer by providing him a guideline to define his system of objectives as detailed as possible in the early stage of product engineering. Based on this information, new ideas can be detected for the current product generation. The activity of "modelling principle solutions" is a necessary step in order to investigate the new drivetrain topologies regarding the fulfilment of the systems of objectives of the systems of objects. This is done in the activity of "validation and verification", whereby this activity can only be performed virtually in this early stage of the product development. The method will be introduced in the prescriptive study, which is presented in section 4. It will be evaluated and tested at the example of hybrid electric vehicle drivetrains but has also the aspiration to be applicable to other drivetrain configurations, such as hydrogen electric vehicle drivetrains.

In Section 5, the results of the comprehensive descriptive studies 1 and 2 will be presented. In the first study, the method will be evaluated and checked for plausibility with test topologies and the results will be compared to topologies of vehicles that are already on the market. In study 2, a customer study will be performed in order to evaluate a development tool that supports the developer in the application of the method. Within this study, the applicability of the method will be evaluated as well.

The initial prescriptive study will give an outlook to further research that might support the developer even better.

\section{New method to synthesize and evaluate hybrid electric vehicle drivetrain topologies}

The new method consists out of four main parts and a fifth downstream part. In a first step, the system of objectives has to be clarified (Albers et al., 2011), in order to define the design space and the rating criteria for the topology evaluation. As the method is applied in a software-based tool, the system of objectives is defined via the input parameters (section 4.1). After the design space is defined, an automated topology synthesis process can identify all mechanical realizable topologies that can be created with a specified number of subcomponents in the drivetrain (section 4.2). Those mechanical realizable topologies are afterwards tested regarding their energy consumption and performance (section 4.3). Based on these test results, the automated evaluation of the topologies, which considers the simulation results and sets them in relation to the defined values in the system of objectives, is performed (section 4.4). The fifth part, which is downstream in the proceeding, checks the synthesized and evaluated topologies for similarity in order to identify potentials to design even more efficient drivetrains for the defined system of objectives (section 4.5). The proceeding of the method is displayed in Figure 1.

\subsection{System of objectives}

As the method is supported by a software based tool, the developer defines the system of objectives in a graphical user interface, which is displayed in Figure 2. In the first step, the vehicle class has to be defined. Afterwards, the intended use of the vehicle for which a powertrain topology is to be found, has to be clarified. Thereby, several categories, such as "sporty", "economic" or "normal" driving usage can be chosen. Furthermore, the region in which the vehicle is to be sold is to be defined in the third step, in order to identify the legal framework conditions for the area of application. In addition, it is to be defined whether a petrol or diesel engine is to be used (step 4) and whether it is a plug-in hybrid or not (step 5). To be able to adapt the rating process to different estimated driving profiles, those have to be specified in step 6. In addition to this information, target values for the performance of the vehicle are also required in order to estimate the power requirements and thus to be able to select the drive units. Based on predecessor models, values for the calculation of driving resistances, such as the drag coefficient or the front surface of the vehicle, are defined in step 7. Based on the input of step 1 to 6 , the developer gets a proposal for each value, which is based on a vehicle class and usage-specific investigation and can be 
adapted to brand specific needs by the developer. In the last step, the developer can start the topology synthesis process and define the design space by limiting the number of drive train components.

\begin{tabular}{|l|}
\hline \multicolumn{1}{|c|}{ Input- } \\
parameters \\
-Vehicle- \\
class and- \\
parameters \\
- Purpose of \\
usage \\
- Target \\
market \\
- Performance \\
require- \\
ments \\
\end{tabular}
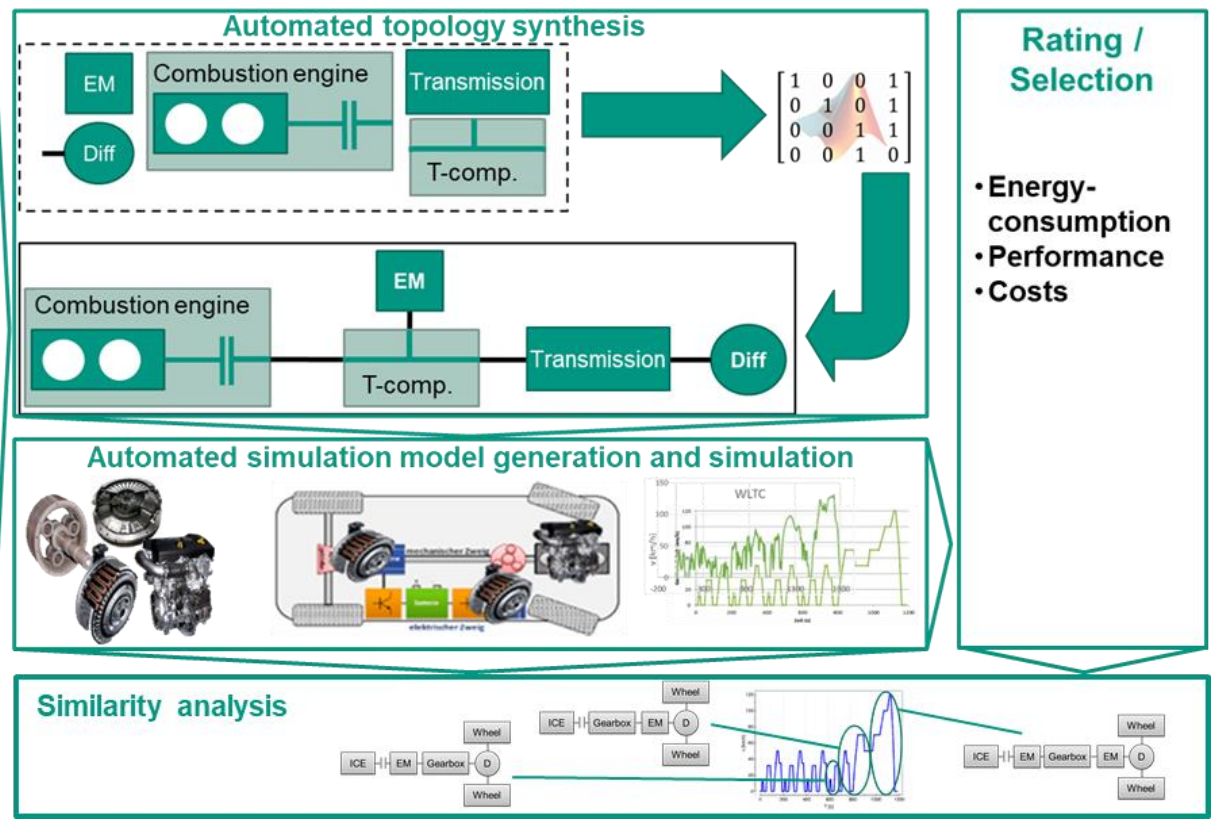

Figure 1. Proceeding of the automated method
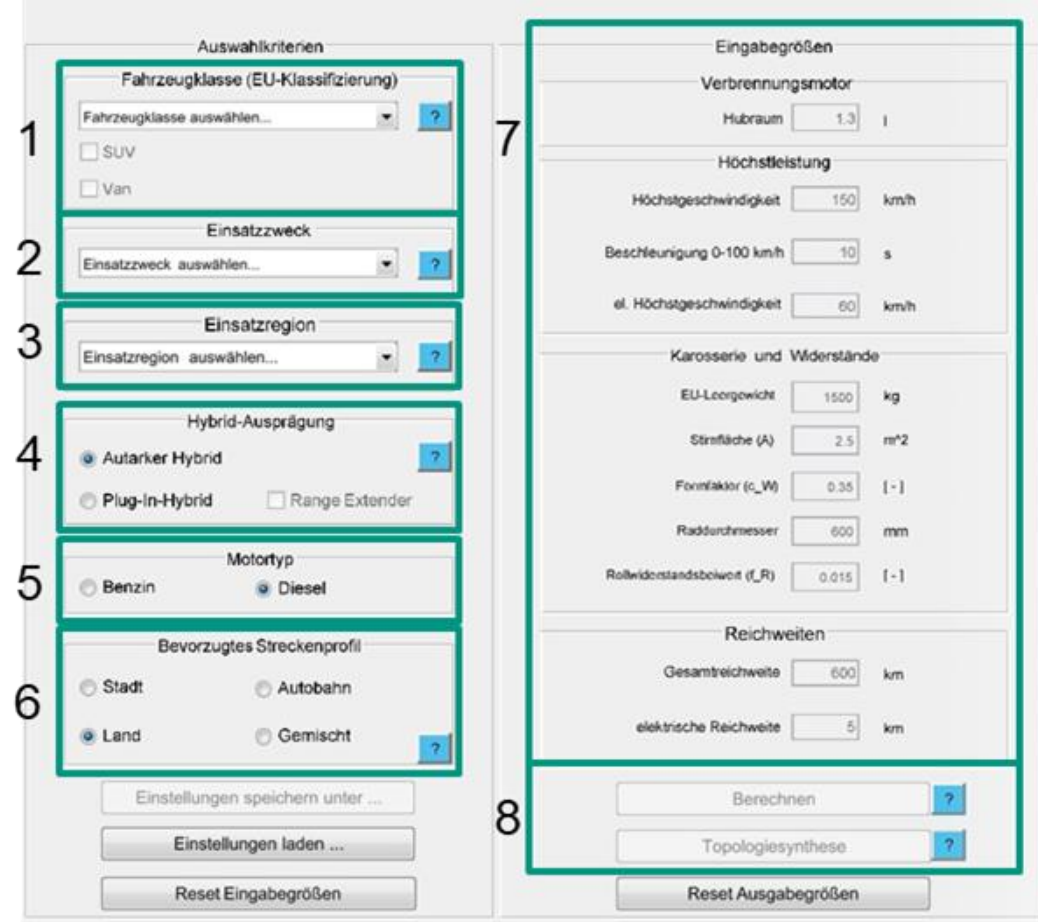

Figure 2. User interface for the definition of the system of objectives

\subsection{Automated topology synthesis}

In order to automate the synthesis of hybrid powertrains, the powertrain topologies are described mathematically. Using graph theory, the powertrains are represented by incidence matrices. Basis for 
the modelling is the definition of the powertrain components as "nodes", in the sense of the graph theory and the mechanical connections between the powertrain components are defined as "edges" (Krische and Röpcke, 2015). The number of mechanical connections of a component defines the number of edges to be connected in the mathematical description. Driving machines, such as the internal combustion engine and the electric motor, are characterized by the fact that they have a mechanical connection and are therefore modelled with one edge. A gearbox has a mechanical input and an output and is therefore modelled as a node with two edges. In order to evaluate the energy efficiency and the performance of the respective topology in the selected use case, a single-track model is implemented which maps the longitudinal dynamics of the vehicle. The residual vehicle model contains the differential, the side shafts and the wheels of the powertrain, which is why the differential is only modelled with one mechanical connection to the powertrain.

Figure 3 shows the schematic representation of a hybrid powertrain. It can be seen that the coaxial connection of an electric machine between combustion engine and transmission cannot be realized by only one mechanical connection to the electric machine.

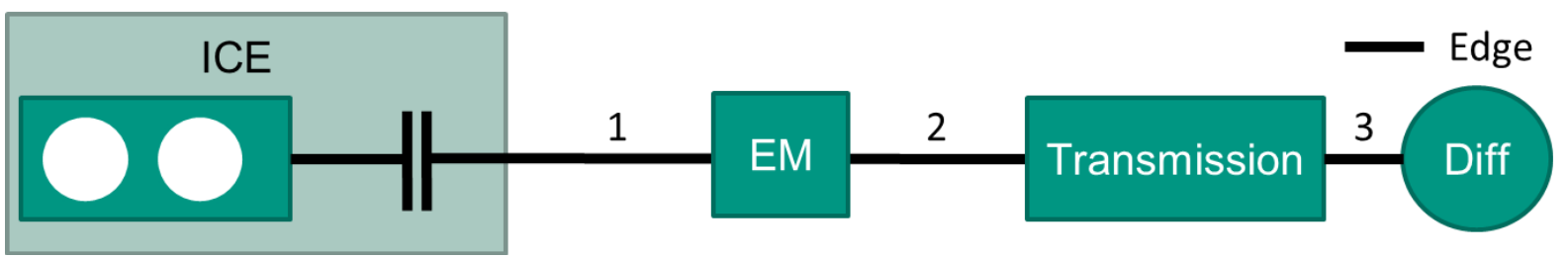

Figure 3. Schematic representation of a P2 hybrid drive train

One possibility would be to introduce two models for an electrical machine, one with one mechanical connection and one with two mechanical connections. In order to avoid errors in the generation, a mechanical interface is introduced that allows the connection of three components. This is hereinafter referred to as the "T-component". Thus, the modelling of the electrical machine with a mechanical interface is sufficient and it is possible to check the correct installation of the electrical machine by means of clear rules. Figure 4 shows a hybrid drive train with a built-in T-component.

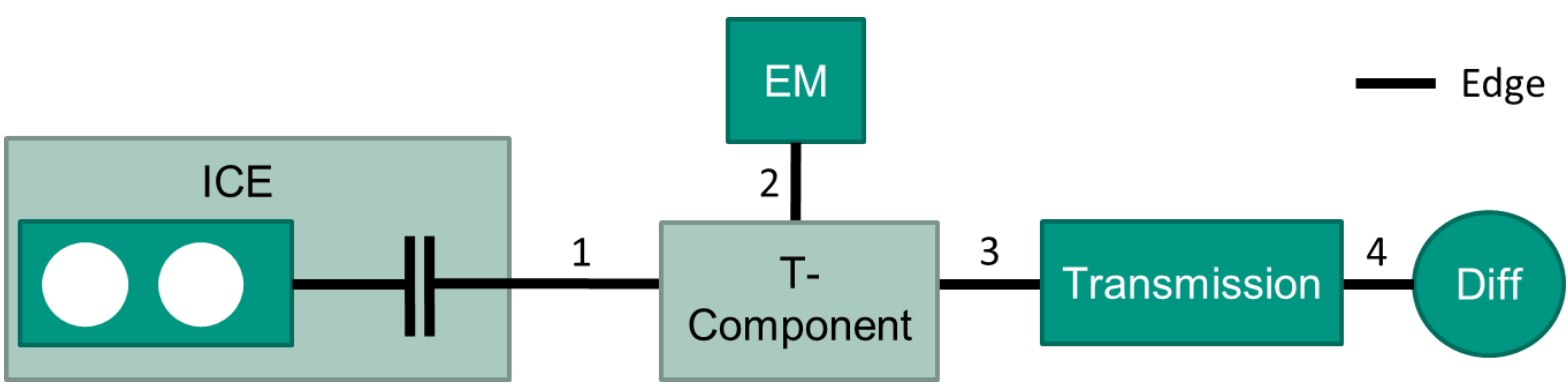

Figure 4. Schematic representation of a P2 hybrid powertrain with T-component

A T-component can stand for several mechanical characteristics in the drive train: On the one hand, lossfree torque addition can be realized via a coaxial connection of the electrical machine. Further mechanical characteristics for torque addition are also possible, such as a gear stage with ratio 1, a chain drive or similar. Within the framework of the simulation, however, a loss-free coaxial connection of the electrical machine during torque addition is assumed. The transfer of the function into the shape of the drive train only takes place after the method and is not relevant for the simulation. On the other hand, a speed addition can take place via a planetary gear. Six different connections of the adjacent components at the planetary gear can be realized, since there are six permutation possibilities at the three connections sun gear, carrier and ring gear. The speed addition cannot be assumed to be lossless due to the losses in the planetary gear.

The topology shown in Figure 4 can be described by an incidence matrix as shown in Table 1 . The mechanical connections between the various components are described in each line. Each line stands for a mechanical connection and therefore has a beginning and an end and therefore exactly two 
entries. The last line defines the last component in the power flow. This line describes the connection to the differential, which is why only one entry is made in the column of the component connected to the differential. A further rule is that the sum of the entries in a column must be equal to the number of mechanical connections of the respective component.

Table 1. Mathematical description of the P2 hybrid topology from Figure 4

\begin{tabular}{|l|c|c|c|c|}
\hline & ICE & EM & Transmission & T-Component \\
\hline Edge 1 & 1 & 0 & 0 & 1 \\
\hline Edge 2 & 0 & 1 & 0 & 1 \\
\hline Edge 3 & 0 & 0 & 1 & 1 \\
\hline Edge 4 & 0 & 0 & 1 & 0 \\
\hline
\end{tabular}

By applying graph theory to the mathematical description of powertrain topologies, constraint satisfaction problems can be described by rules and solved by algorithms (Dechter and Cohen, 2003). Thus, hybrid powertrain topologies can be generated automatically for defined solution spaces and then stored in matrices (Ruoff et al., 2019a).

\subsection{Automated simulation model generation and simulation}

The previously created matrices, which describe the mechanical structure of the powertrain topologies, are then read out and the information used to automatically generate simulation models for each topology. These simulation models can be used to investigate fuel consumption and powertrain performance.

In order to carry out the investigations in relation to the defined system of objectives, the required performance of the combustion engine and the electrical machine(s) must first be determined. For this purpose, the power requirements of the powertrain are taken into account on the basis of the maximum speed and acceleration specifications, taking into account the driving resistances from the system of objectives. Based on the performance requirements, the characteristic maps of the drive machines are selected from a library and the simulation models are parameterised. In addition, the gear ratios for the various gears are calculated and defined in the simulation model. The capacity of the battery is determined on the basis of the electrical range requirements and the battery model is also parametrised accordingly. The remaining vehicle model is parameterized with the data from the system of objectives and the operating strategy is selected depending on the topology class (parallel, serial or power split). At any time, the operating strategy selects the energy-optimal driving mode depending on the state of charge (SoC) and the current power requirement. Before the simulation, a preprocessing is carried out to determine at which driving speed and which acceleration requirement, which combination of gear selection and torque combination of the various drive machines is most suitable from an energetic point of view. Accordingly, at any point in time the driving condition is checked, which requirements from the driving cycle and the driver model are placed on the vehicle and the machines and the transmission are controlled accordingly, as long as the battery SoC is high enough. If the state of charge is too low, the vehicle is driven conventionally.

As part of the fuel consumption simulation, the corresponding driving cycle is stored depending on the system of objectives and driven through with a full and empty battery in order to calculate the fuel consumption in accordance with the standard. In addition, acceleration and maximum speed simulations are carried out, with the help of which the results can be used to check the fulfilment of the requirements in the automated evaluation with regard to performance (Ruoff et al., 2019a).

\subsection{Rating and selection}

In order to enable the developer to identify the most suitable topology for the defined system of objectives, criteria are required that are used for the evaluation of the topologies. The main criteria "energy consumption" and "performance" consist of different sub-criteria. The "costs" for each are determined on the basis of cost models for the drive machines, transmissions and batteries. With the "Analytic Hierarchy Process" (Zimmermann and Gutsche, 1991), the sub-criteria for an overall evaluation are offset 
against a weighting. Since different systems of objectives contain different requirements for the drivetrain, the weighting of the criteria for identifying the overall evaluation must be adapted to the respective system of objectives. For example, the performance of a city vehicle is less important than that of a sports vehicle. Therefore, different weightings are stored in the method, which can be used automatically, depending on the system of objectives, to adjust the result to the respective application. The overall result is output to the developer as a utility value on a scale between 0 and 100 (Ruoff et al., 2018).

The method could end at this point, as the developer gets a suggestion of the best drivetrain topology, which was identified in the defined design space. As there might be even more efficient topologies, which are out of the design space boundaries and could be created by introducing mechanical couplings in the synthesis process, the so called similarity analysis is provided as add-on.

\subsection{Similarity analysis}

The described modelling principle leads to the idea to combine two hybrid topologies with the aim to detect possible positions of a clutch in the drivetrain, in order to make it possible to use different driving modes where different propulsion machines are involved in the drive of the vehicle. Prerequisite for the combination of two hybrid topologies is that both have to have similarities.

It was searched for a solution that leads to an identification of structural similarities, but either ignores functional similarities or even evaluates them as exclusion criteria. So it is not absolutely necessary to consider the simulation results, but it can lead to the fact that beside the synthesizability of two structures also the expressiveness of such an operation can be made recognizable.

When analysing possible combinations, however, not only topologies consisting of the same components and connected in different ways should be identified. To a certain extent, the integration of additional components can also lead to a positive cost-benefit ratio. One of the crucial factors is where they are located. If an additional component is to be placed at the edge of a topology, this is less design effort than if it had to be accommodated centrally, since the entire installation space has to be adapted for this purpose.

Besides the position of the individual components - i.e. integrated in the middle of the topology or exposed - it is also linked to the fact in which components two structures differ. The easiest way to explain this is to give an example: It is assumed that two topologies differ only by one transmission built in at any point. Then a combination is quite simple. A shiftable gear with a gear ratio of one has to be implemented and one can be transferred to the other. If, however, two drive trains are identical except for one planetary gear, exactly the opposite is the case. This means that in both cases it appears in the same place, but the shafts are connected differently. Achieving identical behaviour requires a considerable design effort, for example by inserting several couplings. By installing an additional coupling, exposed components such as the motors can be easily integrated. Therefore, this important aspect has to be taken into consideration in this evaluation as well. The difference between two topologies is ideally calculated according to type, position and connected components of each component.

When all of the drive trains to be tested have been assigned to a similarity value, these still have to be evaluated. The aim is that the user should receive concrete suggestions as to which topologies further investigations or syntheses would be useful. The easiest way to solve this problem is the definition of a distance limit. Above this limit, the topologies are too different to be combined. If their distance is less, they should be further examined. This value could first be determined on the basis of a few examples and then be tested experimentally. Unfortunately, this method is not considering an important aspect: it is not only the constructive effort that is decisive, but also the potential added value of a synthesis. If two structures obtain identical simulation results, it doesn't make much sense to transfer them into each other via clutches, for example, since the resulting vehicle would never be superior to its predecessors. But then again, a high synthesis effort can be justified if there can be achieved a significant increase in performance. In order to assess how promising a combination of two topologies is, a further parameter must be inserted in addition to the distance value. This parameter has to illustrate how differently these different test cycles perform and whether one of the two is already the better choice in all areas.

A lot of data is available after the simulation: The fuel consumption, the state of charge of the battery, the speed driven and many more. These must first be filtered for an evaluation. For a simple distinction as to whether a synthesis can be useful or not, however, it is sufficient to make a 
fundamental statement as to whether both topologies offer advantages over the other. This can be determined on the basis of a number of previously defined parameters: The state of charge of the battery at the end of the driving cycle, the average fuel consumption achieved and the difference between target and actual speed, which are queried after different cycles. The more the two topologies in these areas differ, the more likely it is that a combination will bring benefits.

In order to decide whether or not to recommend a synthesis of two topologies, two criteria are decisive: their feasibility within reasonable effort and their added value in terms of the driving characteristics of the vehicle depicted. The calculated distance values allow the feasibility to be determined while maintaining a limit distance.

The significance of a combination of two topologies is more difficult to assess. A number of characteristics can lead to a synthesis bringing added value. There can be considered many measurable simulation results. If there exists a difference between the topologies across all considered results or if there are clear advantages of one topology in individual areas, the proposal for a synthesis can be made. The regarded properties are: Average fuel consumption, state of charge (SOC) at the end of a driving cycle and the difference between vehicle speed and target speed within a simulation.

Each of these properties are identified and memorized in several driving cycles. These values can be scaled: Each individual result is mapped to a number between zero and one compared to the other topologies. A one is the best of all values in all simulations, a zero is the worst. By scaling all properties for each drive cycle accordingly, a property vector with these values can be defined for each topology. Then, the Euclidean distance of two vectors allows to estimate the extent of the functional differences.

The synthesis is evaluated as meaningful if the Euclidean distance of the property vectors is above a fixed threshold value and if none of the topologies considered dominates the other one. The term dominance means that a particular topology performs better than its counterpart in all considered areas of the simulation. After the meaningfulness has been determined, this information is memorized similarly to the distance values.

For a combination of two topologies, the effort increases proportionally with the distance in between, so the combination of topologies with a greater distance value than required should be avoided (Ruoff et al., 2019b).

\subsection{Interim conclusion}

In this section, a new method is described, that fulfils the needs of the developer in the early stage of PGE in order to identify a hybrid drivetrain for a defined system of objectives. The described research gap can be closed and in order to evaluate the applicability, the method was evaluated and the results of the evaluation are presented in section 5 .

\section{Comprehensive descriptive studies}

In the first study, the aim was to evaluate whether the simulation of the identified topologies has an adequate quality regarding the energy consumption according to test procedures of the new European driving cycle (NEDC) and worldwide harmonized light vehicles test procedure (WLTP). Therefore, topologies were chosen that are also available on the vehicle market. The system of objectives was defined according to the available vehicles. The comparison shown in Table 2 was performed with a BMW3 series hybrid electric vehicle with a parallel hybrid drivetrain architecture. It can be clearly seen that the deviation in consumption in the NEDC is approx. 5\%, whereas the deviation in consumption in the WLTP is much higher at approx. 20\%. On the other hand, however, there is also an increase in electrical energy consumption of approx. 15\%, which is why it can be assumed that the vehicle in the simulation of the WLTP is more frequently driven electrically and therefore has increased electrical consumption and lower fuel consumption. Overall, however, the conclusion can be drawn from the comparison that the total energy consumption in the simulation is in a realistic order of magnitude. Particularly considering the fact that due to the early development stage in which the method is to be used, the drive machines used are only in the same power range and the exact characteristic map of the drive machines of the comparison vehicle is unknown. (Ruoff et al., 2019a) 
Table 2. Comparison of simulation results and vehicle manufacturers specifications

(Ruoff et al., 2019a)

\begin{tabular}{|l|c|c|}
\hline Comparative value & Reference vehicle & $\begin{array}{c}\text { Same vehicle from topology } \\
\text { synthesis }\end{array}$ \\
\hline Consumption NEDC & $1,61 / 100 \mathrm{~km}$ & $1,681 / 100 \mathrm{~km}$ \\
\hline Consumption WLTP & $1,41 / 100 \mathrm{~km}$ & $1,141 / 100 \mathrm{~km}$ \\
\hline Consumption WLTP electric & $15,9 \mathrm{kWh}$ & $17,7 \mathrm{kWh}$ \\
\hline Power ICE & $215 \mathrm{~kW}$ & $214 \mathrm{~kW}$ \\
\hline Power EM & $83 \mathrm{~kW}$ & $86 \mathrm{~kW}$ \\
\hline Weight & $1815 \mathrm{~kg}$ & $1858 \mathrm{~kg}$ \\
\hline
\end{tabular}

In the second study, which was performed with 15 test persons that have experience in drivetrain development, the aim was to evaluate the applicability of the software tool and the developed method. The user interface was rated as understandable and clear. Only instructions for the navigation within the tool were asked for, to be able to operate the tool intuitively. The most important statement that can be made is, that all participants have consensus, that the information that is needed for the synthesis, simulation and rating are asked for within the user interface input parameters. Another important finding is that the test persons were all able to comprehend the output of the tool with the given information to each topology and the topology ranking. The similarity analysis was unanimously recognized as meaningful. All participants beside one could at least recognize the similarity of topologies when there was integrated an additional transmission with the gear ratio of one. When adding a transmission and an electric motor, there are three additional components in the drivetrain, as there is a T-component needed in order to integrate the EM. This similarity was still recognized by half of the participants. The most difficult similarity, when two components of a topology are exchanged and additionally another component is added, which has the largest permitted distance value, was even interpretable by one third of the participants. It was obvious that the participants needed support to identify the similarities, as they did not know the guidelines on which the analysis bases. After a short introduction of an interpretation guideline, the previously mentioned groups could be identified, which recognized different levels of topology pairings as still similar. Overall, it can be concluded, that all participants recognized the application possibilities. They also rated the method to be useful in the early stage of the PGE in order to generate and rate HEV topologies.

\section{Conclusion and outlook}

Based on the investigated research gap, a new method for the support of the developer in the early stage of the PGE of HEVs has been developed and presented in this paper. From the definition of the system of objectives, via the automated synthesis of hybrid drivetrains, the automated simulation, the evaluation and the similarity analysis, the developer is guided in order to identify the most suitable drivetrain for his application. The developed method was implemented in a software tool, which has been evaluated regarding the quality of the synthesis and simulation results and regarding the usability. The investigation has shown that there is only a small deviation in the energy consumption of the simulation compared to the vehicle manufacturer's specifications. With this result it can be said, that the simulation tool is meaningful for the suggestion of the further development of the drivetrain. The applicability and the usability for the tool was also rated very good and the test persons agreed that the use of the method is very helpful to master the complex task of identifying the most suitable drivetrain for a use case.

The described similarity analysis suggests topologies that can be merged in order to get an even more suitable drivetrain for the defined system of objectives. The merging and further investigation has to be done manually by the developer. It should be investigated whether this step can be automated as well, in order to support the developer further and to allow the investigation of several topology pairings, which is quite complex, as the simulation model has to be built manually. 


\section{References}

Albers, A. et al. (2014), "Development of Hybrid-Powertrains by Means of X-in-the-Loop-Approach", Paper presented at Braunschweig Symposium on Hybrid and Electric Vehicles, Vol. 11, 2014, Braunschweig, February 18-19, ITS mobility e.V., Braunschweig, pp. 233-250.

Albers, A. et al. (2016a), "iPeM - integrated Product engineering Model in context of Product Generation Engineering", Paper presented at 26th CIRP Design Conference, Stockholm, Sweden, 2016. https://doi.org/10.1016/j.procir.2016.04.168

Albers, A., Bursac, N. and Rapp, S. (2016b), "PGE-Product Generation Engineering - Case Study of the Dual Mass Flywheel", in D. Marjanović (Ed.), International Design Conference DESIGN 2016, Dubrovnik, Croatia, May 16-19, 2016, The Design Society, Glasgow, pp. 791-800.

Albers, A., Lohmeyer, Q. and Ebel, B. (2011), "Dimensions of objectives in interdisciplinary product development projects", Paper presented at 18th International Conference on Engineering Design 2011 (ICED 2011), August 15-19, Copenhagen, Denmark.

Albers, A. et al. (2017), "Die Frühe Phase der PGE - Produktgenerationsentwicklung", Paper presented at Stuttgarter Symposium für Produktentwicklung 2017, Stuttgart, Germany.

Blessing, L.T.M. and Chakrabarti, A. (2009), DRM, a Design Research Methodology, Springer, London.

Dechter, R. and Cohen, D. (2003), Constraint processing, Morgan Kaufmann Publishers, San Francisco.

Eghtessad, M. et al. (2015), "Antriebsstrangoptimierung von Elektrofahrzeugen", ATZ Automobiltechnische Zeitschrift, No. 09-2015, pp. 78-85.

Haag, A. (2017), Konzepte für effiziente hybride Triebstränge, Springer Fachmedien Wiesbaden.

Krischke, A. and Röpcke, H. (2015), Graphen und Netzwerktheorie: Grundlagen - Methoden - Anwendun-gen; mit zahlreichen Beispielen, Fachbuchverlag Leipzig im Hanser Carl-Hanser-Verlag, München.

Müller, J., Liebold, J. and Danzer, C. (2016), "Leistungsfähiges Hybridgetriebe für zukünftige Plug-inAntriebe", ATZ - Automobiltechnische Zeitschrift, Vol. 118 No. 10, pp. 16-23.

Reuschlé, T. et al. (2016), "Multi-Objective Optimization of Plug-In Hybrid Powertrains - Certification Procedure Sensitivity", in Schäfer, H. (Ed.), Haus der Technik 139: Elektrische Traktions- und Hilfsantriebe für die Elektrifizierung und Hybridisierung von Kraftfahrzeugen, Vol. 139, Expert Verlag, Renningen, pp. 41-59.

Ruoff, S., Busch, M. and Bause, K. (2018), "Evaluation of new hybrid electric vehicle drivetrain topologies", Paper presented at International Electric Vehicle Symposium EVS 31, 30.09.-03.10.2018, Kobe.

Ruoff, S. et al. (2019a), "Methode zur automatisierten Topologiesynthese und Bewertung hybrider Antriebsstränge", in DFX 2019: Proceedings of the 30th Symposium Design for X, 18-19 September 2019, Jesteburg, Germany, 18th-19th September 2019, The Design Society. https://doi.org/10.35199/dfx2019.18

Ruoff, S. et al. (2019b), "Investigation of the necessity of methodical support of the developer to combine the advantages of two hybrid electric topologies in order to increase the number of realizable functions", Paper presented at International Electric Vehicle Symposium EVS 32, 21.-24.05.2019, Lyon.

Silvas, E. et al. (2016), "Review of Optimization Strategies for System-Level Design in Hybrid Electric Vehicles", IEEE Transactions on Vehicular Technology, p. 1.

Silvas, E. et al. (2015), "Functional and Cost-Based Automatic Generatorfor Hybrid Vehicles Topologies", IEEE/ASME Transactions on Mechatronics, Vol. 20 No. 4, pp. 1561-1572.

Sturm, A. et al. (2016), "Powertrain dimensioning of electrified vehicle concepts based on a synthesis", Paper presented at Conference on Future Automotive Technology, 03.-04-05.2016, Fürstenfeldbruck.

Teuschl, G. (2009), "Hybrid- und Elektrofahrzeuge", ATZ - Automobiltechnische Zeitschrift, Vol. 111 No. 6, pp. 434-440.

Voss, B. (2005), "Hybridfahrzeuge", Fachbuch / Haus der Technik, Band, Vol. 52, Expert-Verl., Renningen.

Zimmermann, H.-J. and Gutsche, L. (1991), Multi-Criteria Analyse. Einführung in die Theorie der Entscheidungen bei Mehrfachzielsetzungen, Springer Verlag, Berlin, Heidelberg. 